\title{
Characterization of Series Resistance and Mobility Degradation Parameter and Optimizing Choice of Oxide Thickness in Thin Oxide $\mathbf{N}$-Channel MOSFET
}

\author{
Noureddine Maouhoub and Khalid Rais \\ Laboratoire d'Électronique, d'Instrumentation et de Traitement du Signal, Equipe de Caractérisation des Composants à \\ Semi-Conducteurs, Faculté des Sciences, Université Chouaib Doukkali, BP 20, EL Jadida, Morocco \\ Correspondence should be addressed to Khalid Rais, rais_khalid@hotmail.com \\ Received 28 June 2011; Revised 10 August 2011; Accepted 10 August 2011 \\ Academic Editor: G. Ghibaudo \\ Copyright (๑) 2011 N. Maouhoub and K. Rais. This is an open access article distributed under the Creative Commons Attribution \\ License, which permits unrestricted use, distribution, and reproduction in any medium, provided the original work is properly \\ cited. \\ We present two methods to extract the series resistance and the mobility degradation parameter in short-channel MOSFETs. \\ The principle of the first method is based on the comparison between the exponential model and the classical model of effective \\ mobility and for the second method is based on directly calculating the two parameters by solving a system of two equations \\ obtained by using two different points in strong inversion at small drain bias from the characteristic $I_{d}\left(V_{g}\right)$. The results obtained \\ by these techniques have shown a better agreement with data measurements and allowed in the same time to determine the surface \\ roughness amplitude and its influence on the maximum drain current and give the optimal oxide thickness.
}

\section{Introduction}

Accurate model parameter extraction is crucial for modeling modern MOSFET devices. Extensive work abounds in the literature dedicated to this subject. Free-carrier mobility degradation and source-and-drain series resistance are two parameters of special importance for MOSFET characterization that are particularly cumbersome to extract independently from each other. Both of these parameters produce similar effects on the device's transfer characteristics, $I_{d}\left(V_{g}\right)$, a fact that complicates their accurate extraction.

Several ingenious procedures have been proposed to circumvent this difficulty [1-5]. Another method was proposed to extract these parameters from the drain current versus gate voltage characteristics in the saturation region using several devices of different mask channel lengths [6]. An alternative procedure was recently proposed to extract the source-and-drain series resistance independently of mobility degradation by using bias conditions under which the channel carrier mobility is kept constant [7]. Another recent works presented direct fitting and direct calculating methods in strong inversion and by exploiting the characteristic $I_{d}$ $\left(V_{g}\right)[8,9]$.

In what follows we present a two procedures to be applied to the strong inversion $I_{d}\left(V_{g}\right)$ characteristics of a single transistor, measured at a small drain bias. It is based on the exploiting of exponential model of effective mobility. The procedure has been validated using data from a single experimental short channel device.

The drain current, $I_{d}$, at very small drain bias, can be expressed in terms of intrinsic voltages as

$$
I_{d}=\frac{W}{L} \cdot \mu_{\mathrm{eff}} \cdot C_{\mathrm{ox}} \cdot\left(V_{G}-V_{t}\right) \cdot V_{D}
$$

where $W$ is the channel width, $L$ is the channel length, $C_{\mathrm{ox}}$ is the oxide capacitance, $\mu_{\text {eff }}$ is the effective free-carrier mobility, $V_{G}$ is the intrinsic gate voltage, $V_{D}$ the intrinsic drain voltage, and $V_{t}$ is the threshold voltage. 
If the source-and-drain series resistance is significant, the device's intrinsic gate and drain voltages are

$$
\begin{aligned}
& V_{G}=V_{g}-I_{d} \cdot \frac{R_{\mathrm{sd}}}{2}, \\
& V_{D}=V_{d}-I_{d} \cdot R_{\mathrm{sd}},
\end{aligned}
$$

where $V_{g}$ and $V_{d}$ are the externally applied gate and drain voltages, respectively, and $R_{\mathrm{sd}}$ is the total source-and-drain series resistance.

The model of variation mobility with effective field considers that the attenuation of effective mobility is particularly due to Surface Roughness Scattering [10]. This work generalized all the classical models $[9,11]$ and give a physical meaning to the different used parameters.

The effective mobility is given by the exponential model in strong inversion:

$$
\mu_{\text {eff }}=\mu_{0} \cdot \exp \left(-\theta_{i} \cdot\left(V_{G}-V_{t}\right)\right)
$$

where

$$
\theta_{i}=\frac{\eta \cdot \beta \cdot \Delta \cdot C_{\mathrm{ox}}}{\varepsilon_{\mathrm{si}}}
$$

$\theta_{i}$ is the intrinsic attenuation coefficient of mobility, $\eta$ is constant parameter equal to 0.5 for electrons and 0.33 for holes, $\beta=q / k T$ is the inverse of the thermal potential, $\varepsilon_{\mathrm{si}}$ is the silicon permittivity, $\Delta$ is the surface roughness amplitude, and $\mu_{0}$ represents the low-field mobility.

Assuming that $V_{g}-V_{t} \gg I_{d} \cdot R_{\mathrm{sd}} / 2$, relation (1) becomes

$$
I_{d}=K \cdot \frac{\left(V_{g}-V_{t}\right)}{\exp \left(\theta_{i} \cdot\left(V_{g}-V_{t}\right)\right)+\left(\left(K \cdot R_{\mathrm{sd}}\right) / V_{d}\right) \cdot\left(V_{g}-V_{t}\right)},
$$

where

$$
K=\frac{W}{L} \cdot \mu_{0} \cdot C_{\mathrm{ox}} \cdot V_{d}
$$

Introducing the series resistance effect, the effective mobility becomes

$$
\mu_{\text {eff }}=\frac{1}{\left(1 /\left(\mu_{0} \cdot \exp \left[-\theta_{i} \cdot\left(V_{g}-V_{t}\right)\right]\right)\right)+\theta_{R s d}\left(V_{g}-V_{t}\right)},
$$

where

$$
\theta_{R \mathrm{sd}}=\frac{K \cdot R_{\mathrm{sd}}}{V_{d}}
$$

The transconductance is given by

$$
g_{m}=\frac{d I_{d}}{d V_{g}}=K \cdot \frac{\left(1-\theta_{i} \cdot\left(V_{g}-V_{t}\right)\right) \exp \left(\theta_{i} \cdot\left(V_{g}-V_{t}\right)\right)}{\left[\exp \left(\theta_{i} \cdot\left(V_{g}-V_{t}\right)\right)+\theta_{R s d} \cdot\left(V_{g}-V_{t}\right)\right]^{2}} .
$$

\section{First Method}

According to the classical model [10], and for low fields, the comparison of the first-order development of the exponential term in relation (3) gives the relation between the intrinsic coefficient attenuation mobility in exponential model and its value extrinsic $\theta_{e}$ in the classical model:

$$
\theta_{e}=\theta_{i}+\theta_{R s \mathrm{~d}}
$$

The values of $K$ and $\theta_{e}$ are determined, respectively, by the slope in the plot of $Y_{0}\left(V_{g}\right)=I_{d} / \sqrt{g_{m}}$ and $H_{0}\left(V_{g}\right)=1 / \sqrt{g_{m}}$ which are straight line at relative low field.

For thin oxide and at high field, the characteristic $Y\left(V_{g}\right)=I_{d} / \sqrt{g_{m}}$, which has the advantage to be independent of series resistance, presents a deviation with regard to $Y_{0}\left(V_{g}\right)$.

We note that $g_{m}$ can reach the value zero at $V_{g m}$ :

$$
\theta_{i}=\frac{1}{\left(V_{g m}-V_{t}\right)}
$$

Experimentally we use the device below this value, meanwhile we use the characteristic $\left(Y\left(V_{g}\right) / Y_{0}\left(V_{g}\right)-1\right)$ versus $V_{g}$. The value at ten per; cent of this characteristic is corresponding to the critical value $\left(V_{g m}-V_{t}\right) / 2=1 / 2 \theta_{i}$; combining (11) with relations (3) and (10) we obtain the surface roughness amplitude $\Delta$ and series resistance $R_{\mathrm{sd}}$, respectively.

\section{Second Method}

The procedure involves first extracting the expression $\exp \left(\theta_{i}\left(V_{g}-V_{t}\right)\right)$ from the drain current equation and using the function $Y=I_{d} / \sqrt{g_{m}}$.

Setting $x=V_{g}-V_{t}$, and after some mathematical manipulation we obtain the following expression:

$$
\frac{Y^{-2} \cdot x}{1-\theta_{i} \cdot x}=\frac{1}{I_{d}}-\frac{R_{\mathrm{sd}}}{V_{d}}
$$

Secondly, we choose two points in the characteristic $I_{d}\left(V_{g}\right)$ in strong inversion $V_{g 1}$ and $V_{g 2}\left(x_{1}\right.$ and $\left.x_{2}\right)$, and we solve the following system:

$$
\begin{aligned}
& \frac{Y^{-2}\left(V_{g 1}\right) \cdot x_{1}}{1-\theta_{i} \cdot x_{1}}=\frac{1}{I_{d}\left(V_{g 1}\right)}-\frac{R_{\mathrm{sd}}}{V_{d}}, \\
& \frac{Y^{-2}\left(V_{g 2}\right) \cdot x_{2}}{1-\theta_{i} \cdot x_{2}}=\frac{1}{I_{d}\left(V_{g 2}\right)}-\frac{R_{\mathrm{sd}}}{V_{d}} .
\end{aligned}
$$




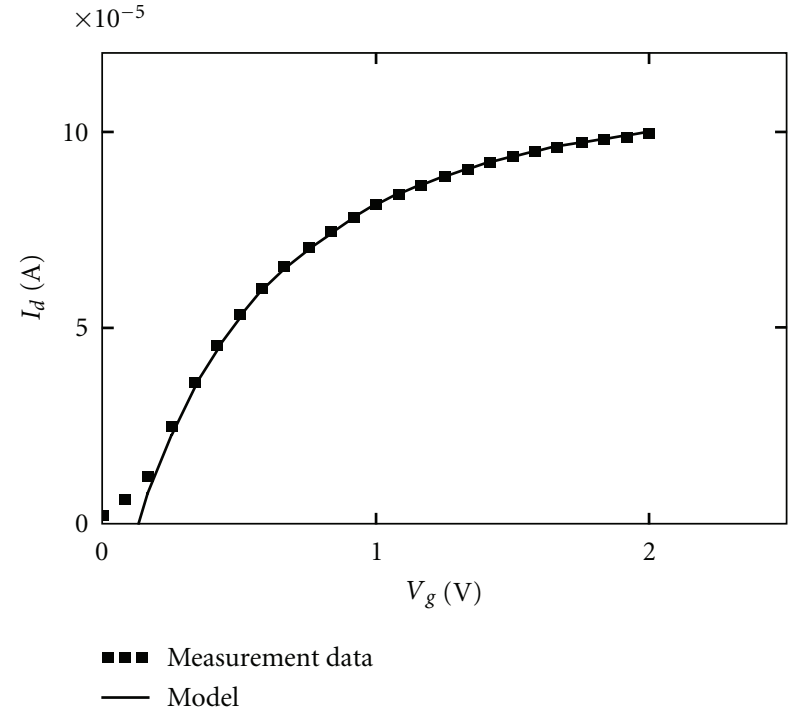

FIgURE 1: Transfer characteristics (symbols). Also shown is the corresponding model (continuous line) obtained from the drain current model using the calculated parameters (Table 1).

Determining the parameter $\theta$ is done using the subtraction of the two system equations; we obtain a second order equation whose appropriate solution is:

$$
\begin{aligned}
& \theta_{i} \\
& \quad+\frac{\left(B \cdot x_{1}+C \cdot x_{2}+C \cdot x_{1}-A \cdot x_{2}\right)}{2 \cdot C \cdot x_{1} \cdot x_{2}} \\
& +\frac{\sqrt{\left(B \cdot x_{1}+C \cdot x_{2}+C \cdot x_{1}-A \cdot x_{2}\right)^{2}+4 \cdot x_{1} \cdot x_{2} \cdot(A-B-C)}}{2 \cdot C \cdot x_{1} \cdot x_{2}},
\end{aligned}
$$

where

$$
\begin{gathered}
A=Y^{-2}\left(V_{g 1}\right) \cdot x_{1}, \\
B=Y^{-2}\left(V_{g 2}\right) \cdot x_{2}, \\
C=\frac{1}{I_{d}\left(V_{g 1}\right)}-\frac{1}{I_{d}\left(V_{g 2}\right)} .
\end{gathered}
$$

The series resistance expression can be written using one of the two system equations:

$$
R_{\mathrm{sd}}=V_{d} \cdot\left(\frac{1}{I_{d}\left(V_{g 2}\right)}-\frac{Y^{-2}\left(V_{g 2}\right) \cdot x_{2}}{1-\theta_{i} \cdot x_{2}}\right) .
$$

The choice of $V_{g 1}$ and $V_{g 2}$ is determined in mobility degradation effect zone.

\section{Results and Discussion}

In order to validate the previous procedures, the device used in this work has the parameters: channel width $W=4 \mu \mathrm{m}$,

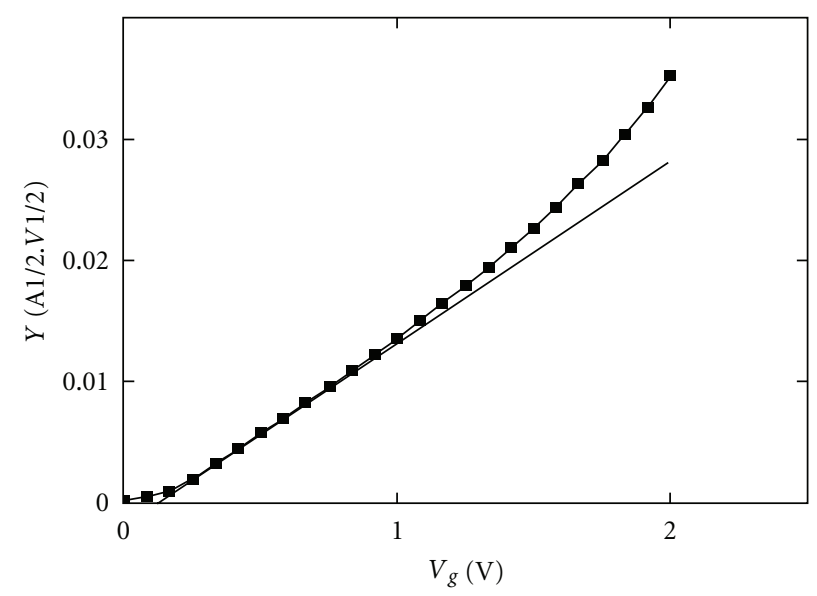

FIGURE 2: Characteristic $Y$ versus gate voltage.

TABLE 1: Values of the different extracted parameters.

\begin{tabular}{lccc}
\hline Parameter & $\theta_{i}(1 / \mathrm{V})$ & $R_{\text {sd }}(\Omega)$ & $\Delta(\mathrm{nm})$ \\
\hline First method & 0.363 & 107.53 & 0.31 \\
Second method & 0.36 & 108.8 & 0.28 \\
\hline
\end{tabular}

gate oxide thickness $t_{\mathrm{ox}}=5 \mathrm{~nm}$, channel length $L=0.1 \mu \mathrm{m}$, and channel doping $\mathrm{Na}=10^{16} \mathrm{~cm}^{-3}$.

The transfer characteristics were measured at a small drain voltage: $V_{d}=20 \mathrm{mV}$. The application of our model with calculated parameters (Table 1) shows a good agreement with experimental data (Figure 1).

In relatively weak field we use model (1) as an approximation of our model; we plot this function $Y\left(V_{g}\right)$. The parameter $V_{t}$ can be extracted from the $V_{g}$ axis intercept of the observed straight lines of $Y$ versus $V_{g}$ plot (Figure 2).

Figure 3 shows the characteristic $\left(Y\left(V_{g}\right) / Y_{0}\left(V_{g}\right)-1\right)$ versus $V_{g}$ which allows determining the intrinsic attenuation parameter.

\section{Optimization of Drain Current}

At $V_{g}=V_{g m}$, the drain current is maximal, its value is

$$
I_{d \max }=\frac{V_{d}}{R_{\mathrm{ch} \min }+R_{\mathrm{sd}}},
$$

where $R_{\mathrm{ch} \text { min }}$ is the minimal resistance of channel:

$$
R_{\text {ch min }}=\frac{\Delta \beta \eta L}{W \mu_{0} \varepsilon_{\mathrm{si}}} \cdot e .
$$

We note its independence of the oxide thickness and its proportionality of the surface roughness amplitude and of the channel length.

On the other hand, if we plot $I_{d}$ versus the oxide thickness $t_{\text {ox }}$ for a given gate voltage $V_{g}$ (Figure 4), we can find the optimal oxide thickness. In fact, for very thin oxide the collisions on surface roughness predominate, and for thick oxide the inversion charge decreases, and consequently the drain current. 


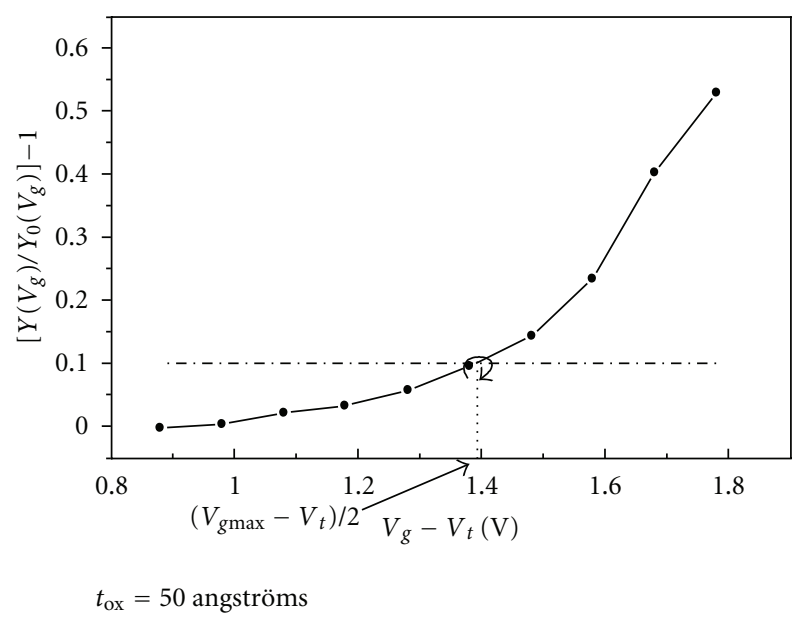

FIgURE 3: Characteristic $Y\left(V_{g}\right)$ to $Y_{0}\left(V_{g}\right)$ ratio versus gate voltage.



Figure 4: Drain current versus oxide thickness for $V_{g}=1.5 \mathrm{~V}$.

\section{Conclusion}

We have presented two procedures that permit us to extract the mobility degradation factor, the series resistance, and the surface roughness thickness of MOSFET using the exponential model of mobility. The procedures are simple and accurate and based on calculating the model parameters using the experimental data measured in strong inversion at small drain bias. The exponential model of mobility allows also us to optimize the oxide thickness for the transistor.

\section{References}

[1] C. L. Lou, W. K. Chim, D. S. H. Chan, and Y. Pan, "A novel single-device DC method for extraction of the effective mobility and source-drain resistances of fresh and hot-carrier degraded drain-engineered MOSFET's," IEEE Transactions on Electron Devices, vol. 45, no. 6, pp. 1317-1323, 1998.

[2] G. M. Lim, Y. C. Kim, D. J. Kim, Y. W. Park, and D. M. Kim, "Additional resistance method for extraction of separated nonlinear parasitic resistances and effective mobility in MOSFET's," Electronics Letters, vol. 36, no. 14, pp. 12331234, 2000.

[3] C. S. Ho, Y. C. Lo, Y. H. Chang, and J. J. Liou, "Determination of gate-bias dependent source/drain series resistance and effective channel length for advanced MOS devices," SolidState Electronics, vol. 50, no. 11-12, pp. 1774-1779, 2006.

[4] P. R. Karlsson and K. O. Jeppson, "An efficient method for determining threshold voltage, series resistance and effective geometry of MOS transistors," IEEE Transactions on Semiconductor Manufacturing, vol. 9, no. 2, pp. 215-222, 1996.

[5] C.-Y. Lu and J. A. Cooper Jr., "A new constant-current technique for MOSFET parameter extraction," Solid-State Electronics, vol. 49, no. 3, pp. 351-356, 2005.

[6] F. J. García-Sánchez, A. Ortiz-Conde, A. Cerdeira, M. Estrada, D. Flandre, and J. J. Liou, "A method to extract mobility degradation and total series resistance of fully-depleted SOI MOSFET's," IEEE Transactions on Electron Devices, vol. 49, no. 1, pp. 82-88, 2002.

[7] D.-W. Lin, M.-L. Cheng, S.-W. Wang, C.-C. Wu, and M.J. Chen, "A constant-mobility method to enable MOSFET series-resistance extraction," IEEE Electron Device Letters, vol. 28, no. 12, pp. 1132-1134, 2007.

[8] A. Ortiz-Conde, F. J. García-Sánchez, J. Muci et al., "Indirect fitting procedure to separate the effects of mobility degradation and source-and-drain resistance in MOSFET parameter extraction," Microelectronics Reliability, vol. 49, no. 7, pp. 689692, 2009.

[9] J. Muci, D. C. L. Muñoz, Á. D. L. Rey et al., "A new integrationbased procedure to separately extract series resistance and mobility degradation in MOSFET's," Semiconductor Science and Technology, vol. 24, no. 10, 105015, 6 pages, 2009.

[10] A. El Abbassi, Y. Amhouche, E. Bendada, R. Rmaily, and K. Raïs, "Characterization of series resistances and mobility attenuation phenomena in short channel MOS transistors," Active and Passive Electronic Components, vol. 24, no. 1, pp. 13-22, 2001.

[11] G. Ghibaudo and F. Balestra, "Modelling of ohmic MOSFET operation at very low temperature," Solid State Electronics, vol. 31, no. 1, pp. 105-108, 1988. 

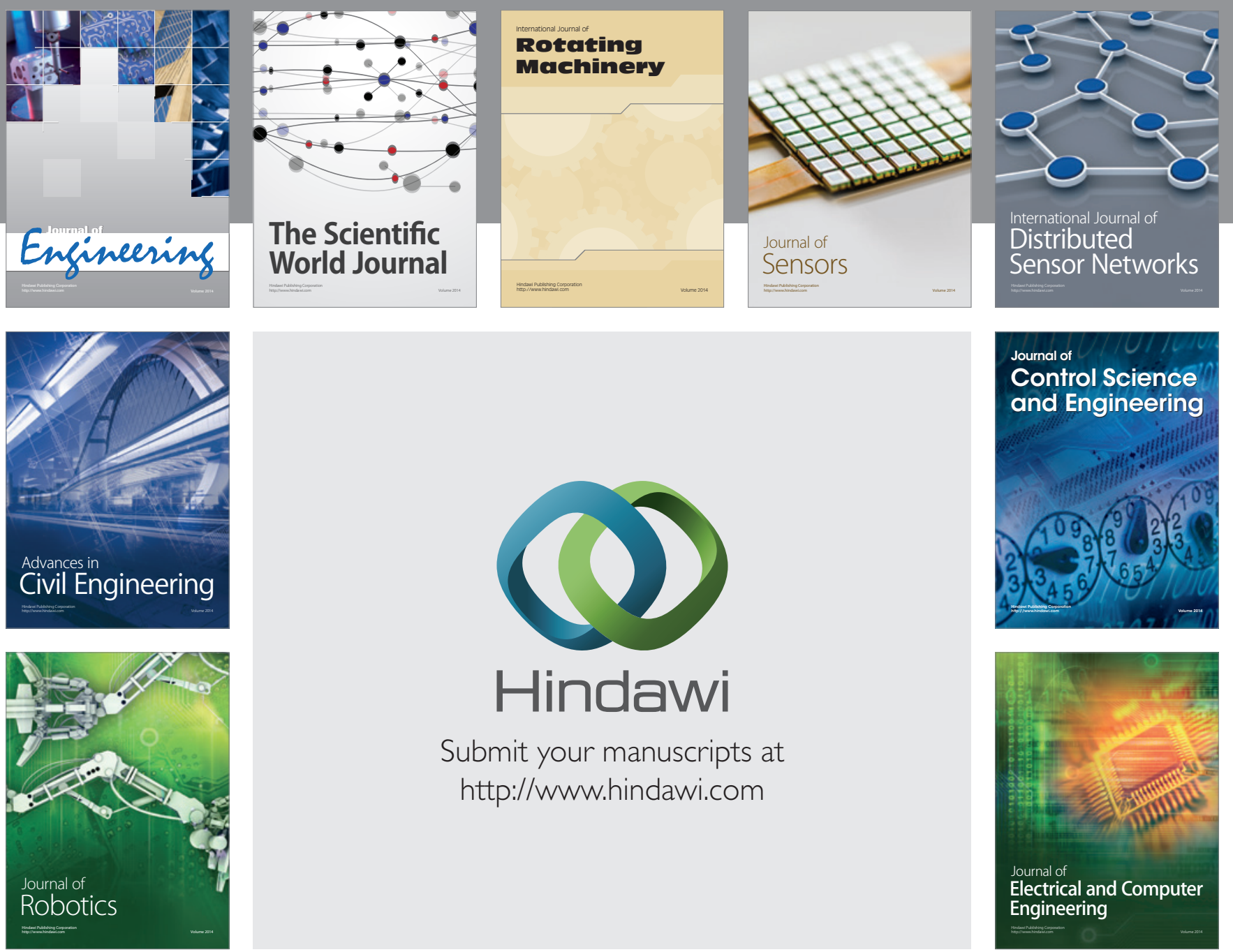

Submit your manuscripts at

http://www.hindawi.com
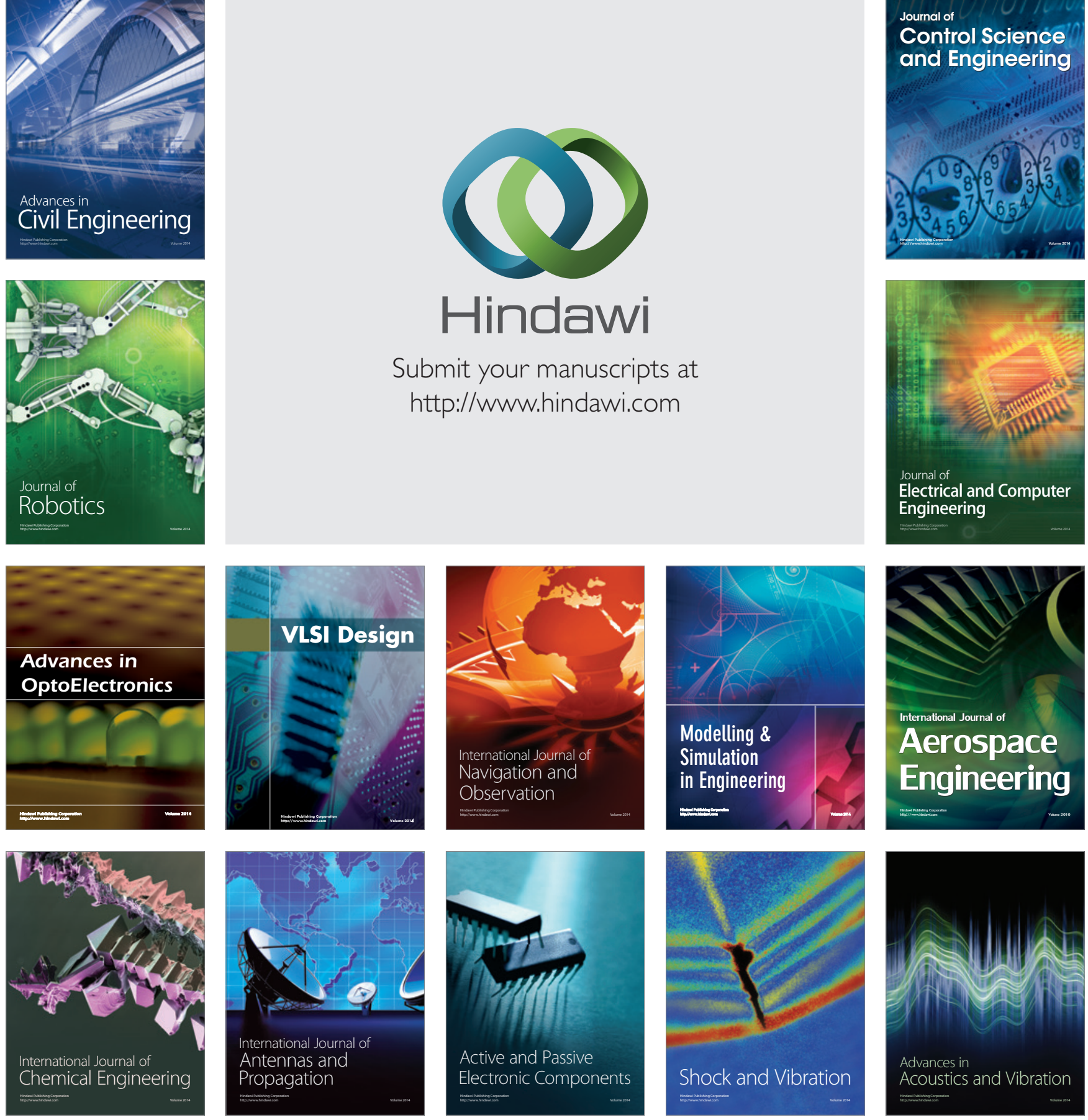